\title{
BILATERAL RANDOM WALKS ON COMPACT SEMIGROUPS 1
}

\author{
A. MUKHERJEA AND N. A. TSERPES
}

ABSTRACT. Let $\mu$ be a regular Borel probability measure with support $F$ on a compact semigroup $S$. Let $X_{0}, X_{ \pm 1}, X_{ \pm 2}, \cdots$ be a sequence of independent random variables with values in $S$, having identical distribution $P\left(X_{n} \in B\right)=\mu(B)$. The random walk $W_{n}=X_{-n} \cdots X_{-1} X_{0} X_{1} \cdots X_{n}$ is studied in this paper. Let $D$ be the closed semigroup generated by $F$ and let $K$ be the kemel of $D$. An element $x \in D$ is called recurrent iff $P_{x}\left(W_{n} \in N_{x}\right.$ i.o. $)=1$ for every open neighborhood $N_{x}$ of $x$. We prove: $x$ is essential for $W_{n}$ if and only if $x \in K$ if and only if $x$ is recurrent if and only if $\Sigma P_{x}\left(W_{n} \in N_{x}\right)=\Sigma \mu^{n} *\left[\mu^{n}\left(x^{-1} \cdot\right)\right]\left(N_{x}\right)=\infty$ for every $N_{x}$. Moreover all states in $K$ are recurrent positive. These results extend results of the authors for the unilateral random walks (using different methods) and recent results of Larisse for the discrete case.

1. Let $S$ be a compact semigroup, i.e., a compact Hausdorff space which is algebraically a semigroup with jointly continuous multiplication and let $S$ have a countable basis. (See Concluding remarks on the blanket assumption of 2 nd countability and how it can be removed.) Let $\mu$ be a regular probability measure defined on the Borel subsets of $S$ whose support is denoted by $F$. Let $D=\overline{U F^{n}}$, the closed subsemigroup generated by $F$, and let $K$ be the kernel of $D$. The measure $\mu$ induces various random walks on $D$ among which the principal ones are the right, left and bilateral (symmetric) random walks induced by the probability transition functions (respectively)

$$
P^{n}(x, B)=\left\{\begin{array}{l}
P_{r}^{n}(x, B) \equiv \mu^{n}\left(x^{-1} B\right) \equiv{ }_{x}(\mu)^{n}(B), \\
P_{l}^{n}(x, B) \equiv \mu^{n}\left(B x^{-1}\right) \equiv\left(\mu^{n}\right){ }_{x}(B), \\
P_{b}^{n}(x, B) \equiv \mu^{n} \times \mu^{n}\left\{(a, b) ; a x b \in B, a, b \in F^{n}\right\} \equiv \mu^{n} *_{x}\left(\mu^{n}\right)(B)
\end{array}\right.
$$

for $n=1,2, \cdots, 2$ where $\mu^{n}$ denotes the $n$th convolution (*) power of $\mu$. For

Received by the editors May 17, 1973 and, in revised form, November 5, 1973. AMS (MOS) subject classifications (1970). Primary 60G50, 60J15, 43A05.

Key words and phrases. Compact topological semigroup, kernel of a compact semigroup, bilateral and unilateral recurrent random walks on semigroups induced by probability measures.

1 Under a USF Research Council Grant.

2 The last equality is proven in Lemma 2.1. 
probabilities $\mu, \nu$,

$$
\mu * \nu(B) \equiv \int \mu\left(B x^{-1}\right) \nu(d x)=\int \nu\left(x^{-1} B\right) \mu(d x) .
$$

These random walks can be represented as sequences of products of independent identically distributed (according to $\mu$ ) random variables $X_{i}=$ the coordinate projections of the product measure space $\left(D^{\infty} \equiv \Pi_{-\infty}^{\infty} D_{i}, P\right), D_{i}=$ $D$, such that

$$
\begin{aligned}
& Z_{n} \text { (right) }=X_{1} X_{2} \cdots X_{n} ; S_{n} \text { (left) }=X_{n} X_{n-1} \cdots X_{1} ; 3 \\
& W_{n} \text { (bilateral, symmetric) }=X_{-n} X_{-n+1} \cdots X_{-1} X_{0} X_{1} \cdots X_{n-1} X_{n} .
\end{aligned}
$$

For the significance of the bilateral random walk, we observe that when $D$ is discrete, the transition matrix for $W_{n}$ is the (commutative) product of the transition matrices for $Z_{n}$ and $S_{n}$. In the continuous case we have

$$
P_{b}(x, B)=\int P_{r}(z, B) P_{l}(x, d z)=\int P_{l}(z, B) P_{r}(x, d z) .
$$

A state $x \in D$ is called recurrent in the right (left) random walk if $P_{x}\left(Z_{n}\left(S_{n}\right) \epsilon\right.$ $N_{x}$ infinitely often (i.o.)) $=1$ for every open neighborhood $N_{x}$ of $x$. Similarly $x \in D$ is called recurrent in the bilateral walk if

$$
P\left(W_{n} \in N_{x} \text { i.o. } \mid W_{0}=X_{0}=x\right)=P_{x}\left(W_{n} \in N_{x} \text { i.o. }\right)=1 \text { for every } N_{x} \text {. }
$$

For $x, y \in D$, we say that $x$ leads to $y(x \rightarrow y)$ in the bilateral walk if $y \epsilon$ $\overline{U F^{n} x F^{n}}$ i.e., for each $N_{y}$ of $y$ there is an $n$ such that $P_{b}^{n}\left(x, N_{y}\right)$ is positive. We say that $x$ and $y$ communicate if $x \rightarrow y$ and $y \rightarrow x$.

In [6] and [2] we studied the unilateral random walks on completely simple and compact semigroups respectively. In the compact case [2] we showed that $Z_{n}$ and $S_{n}$ are always recurrent with their recurrent states forming precisely the kernel (= minimal ideal) $K$ of $D$. Moreover recurrence was characterized in terms of divergence of the series $\Sigma \mu^{n}\left(x^{-1} N_{x}\right)=\Sigma P_{r}^{n}\left(x, N_{x}\right)=$ $\infty$ for all open sets $N_{x}$ containing $x, x$ an element of $D$ (which is equivalent to $x$ being recurrent in $K$ ). This last result of [2] is the only major fact we are using in the present paper, while the methods employed in the present paper are different from those of [2] and [6]. Different methods were needed to handle the bilateral case since the methods used in [2] for the unilateral walk do not seem directly applicable to the symmetric case, the main reason being the different form of $n$-transition probability function for $W_{n}$. It turns out that again $x$ is essential (i.e., $x \rightarrow y$ implies $y \rightarrow x$ ) for $W_{n}$ if and only

3 One of the reasons for the assumption of 2 nd countability is to insure that the $Z_{n}$ 's are measurable as functions on $\left(D^{\infty}, P\right)$. 
if $x \in K$ and that the set of recurrent states for $W_{n}$ is (as in the unilateral case) precisely the kernel $K$ (the states in $K$ being recurrent positive). This was proved by Larisse [1] under the hypotheses that there is a recurrent state and $D$ is discrete. We conjecture that for locally compact completely simple $S=E \times G \times F$ (with $G$ a group), the above result is also true i.e., that $x$ is recurrent in the unilateral if and only if $x$ is recurrent in the bilateral walk, in which case $D$ is a lso completely simple.

Throughout $N_{x} \equiv N(x)$ (or $N_{1}(x), N_{2}(x)$ etc.) will denote open neighborhoods of $x$.

2. Lemma 2.1. Let $B$ be a Borel subset of $D$. Then $A=\{(y, z) ; y x z \in B$, $y, z \in D\}$ is (Borel) measurable and $\mu^{n} \times \mu^{n}(A)=\mu^{n} *{ }_{x}\left(\mu^{n}\right)(B)=P_{b}^{n}(x, B)$.

Proof. If $\phi(y, z)=y x z$, then $\phi$ is a continuous mapping from $D \times D$ into $D$ so that $A=\phi^{-1}(B)$ is also measurable in $D \times D$ (by the second countability of $D$. .) Now by Fubini's theorem,

$$
\mu^{n} \times \mu^{n}(A)=\int \mu^{n}\left(A_{y}\right) \mu^{n}(d y)=\int \mu^{n}\left(x^{-1} y^{-1} B\right) \mu^{n}(d y)=\mu^{n} *{ }_{x}\left(\mu^{n}\right)(B) .
$$

Lemma 2.2. Let $U$ be an open set in $D$. Then the mapping $x \rightarrow \mu^{n} *{ }_{x}\left(\mu^{n}\right)(U)$ is lower semicontinuous.

Proof. Let $y_{k} \rightarrow y$. Then by Fatou's theorem,

$$
\begin{aligned}
\lim _{k \rightarrow \infty} \int \mu^{n}\left(y_{k}^{-1} z^{-1} U\right) \mu^{n}(d z) & \geq \int \underline{\lim } \mu^{n}\left(y_{k}^{-1} z^{-1} U\right) \mu^{n}(d z) \\
& \geq \int \mu^{n}\left(y^{-1} z^{-1} U\right) \mu^{n}(d z)
\end{aligned}
$$

since $y \rightarrow \mu^{n}\left(y^{-1} z^{-1} U\right)$ is a lower semicontinuous function by $[4, \mathrm{p} .130]$.

Lemma 2.3. If $x \in K(=$ the kernel of $D)$, then $x \rightarrow x$ (with respect to the bilateral walk).

Proof. Let $x \in K$. Since $K$ is completely simple, $4 x x=x D x$ is a group and $x \in x K x$. Let $e$ be the identity of $x K x$ and let $y \in x K x$ such that $x y=$ $y x=e$. Since $x y e x=x$, given a neighborhood $N(x)$ of $x$, there exists $N_{1}(e)$ such that $x y N_{1}(e) N_{1}(e) x \subset N(x)$. Since $e \in x D, N_{1}(e) \cap x F^{k} \neq \varnothing$ for some

4 The kernel $K$ of a compact semigroup is always nonempty and completely simple and $x K x(\forall x \in K)$ is a group. For the se and other facts about completely simple semigroups, we refer to Clifford and Preston, The algebraic theory of semigroups. I, II, Math. Surveys No. 7, Amer. Math. Soc., Providence, R. I., 1961 and 1967 resp. MR 24 \#A2627; 36 \#1558. 
$k$, and so $x y N_{1}(e) N_{1}(e) x \cap x y x F^{k} x F^{k} x \neq \varnothing$. Therefore $N(x) \cap\left(x F^{k} x F^{k} x\right) \neq$ $\not$. Therefore there is $w \in F^{k} x F^{k}$ such that $x w x \in N(x)$. Now there is $N_{2}(x)$ such that $N_{2}(x) w N_{2}(x) \subset N(x)$. Also $N_{2}(x) \cap F^{m} \neq \varnothing$ for some $m$. Hence $N(x) \cap F^{m+k} x F^{m+k} \neq \varnothing$. This means that $x \in \overline{\bigcup_{n=1}^{\infty} F^{n} x F^{n}}$ or $x \rightarrow x$. Q.E.D.

Lemma 2.4. $x$ is an essential state (bilateral walk) if and only if $x \in K$.

Proof. First, suppose $x$ is essential in the bilateral walk. Then for all $y \in \overline{\bigcup_{n=1}^{\infty} F^{n} x F^{n}}, x \in \overline{\bigcup_{n=1}^{\infty} F^{n} y F^{n}}$. Clearly $x \in D x D$ (= an ideal of $D$ ). Let $z \in D x D=\overline{\bigcup_{n, m} F^{n} x F^{m}}$. Then there exist $z_{n}=f_{n} x f_{m_{n}}\left(f_{n} \in F^{n}, f_{m_{n}} \in F^{m_{n}}\right)$ such that $z_{n} \rightarrow z$. If $m>n$, let $f_{m_{n}-n} \in F^{m_{n}-n}$. Then $f_{m_{n}-n} f_{n} x f_{m_{n}} \in F^{m_{n}} x F^{m_{n}}$ and so $x \in \overline{\bigcup_{k=1}^{\infty} F^{k} f_{m_{n}-n} f_{n} x f_{m_{n}} F^{k}} \subset D z_{n} D$ for all $n$. Since $D$ is compact, $x \in D z D$. This means that $D x D \subset D z D \subset D x D$ for all $z \in D x D$ or $D x D$ is the kernel $K$ of $D$. Hence $x \in K$.

Second, let $w \in K=$ the kernel of $D$. We now show that $w$ is essential in two steps.

Step I. Let $x, y \in e K e, e$ being some idempotent of $K$ ( $e$ Ke being a group with identity $e$ ) such that $x y=e$. Given $N(y)$, there exists $N_{1}(e)$ such that $N(y) \supset y N_{1}(e) N_{1}(e) e$. Now $e \in x D$ so that $N_{1}(e) \cap x F^{n} \neq \varnothing$ for some $n$ or $N(y) \cap y x F^{n} x F^{n} e \neq \varnothing$. This means that $N(y) \cap F^{m} x F^{m} \neq \varnothing$ for some $m$, or $y \in \overline{\bigcup_{k=1}^{\infty} F^{k} x F^{k}}$, or $x \rightarrow y$. Similarly we show that $y \rightarrow x$.

Step II. Let $w \in K$. Then if $e$ is the identity of the group $w K w$, we have $e K e=w K w$. Let $y=f_{k} w g_{k}, f_{k} \in F^{k}, g_{k} \in F^{k}$. Then eye $=\left(e f_{k} e\right) w\left(e g_{k} e\right) C$ $\overline{\bigcup_{n=1}^{\infty} F^{n} w F^{n}}$. Now $w=\left(e f_{k} e\right)^{-1}(e y e)\left(e g_{k} e\right)^{-1}$ (the inverse being taken in $e K e)$ or $w^{-1}=\left(e g_{k} e\right)(e y e)^{-1}\left(e f_{k} e\right)$ which means that $(e y e)^{-1} \rightarrow w^{-1}$. Now

$$
\text { eye } \underset{\text { (by Step I) }}{\longrightarrow}(\text { eye })^{-1} \longrightarrow w^{-1} \underset{\text { (by Step I) }}{\longrightarrow} w \text {, }
$$

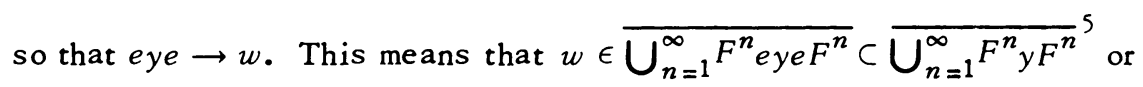
$y \rightarrow w$ (for every $y \in \bigcup_{n=1}^{\infty} F^{n} w F^{n}$ ).

Now let $z \in \overline{\bigcup_{n=1}^{\infty} F^{n} w F^{n}}$. Then there exist $y_{a} \in \bigcup_{k=1}^{\infty} F^{n} w F^{n}$ such

${ }^{5} e \in \overline{\bigcup_{k} F^{k}} \Rightarrow \exists z_{a} \in \bigcup_{k} F^{k}, z_{\alpha}$ converging to $e$ so that $z_{a} y z_{a}$ converge to eye or eye $\epsilon \overline{\bigcup_{k} F^{k} y F^{k}}$. Hence for all $n, F^{n}$ eye $F^{n} \overline{\bigcup_{k} F^{k} y F^{k}} F^{n} \subset$ $\overline{F^{n}\left(\bigcup_{k} F^{k} F^{k}\right) F^{n}} \subset \overline{\bigcup_{k} F^{k+n} y F^{k+n}} \subset \overline{\bigcup_{k} F^{k} F^{k}}$. 
that $y_{\alpha} \rightarrow z$ and $w \in \overline{\bigcup_{n=1}^{\infty} F^{n} y_{\alpha} F^{n}}$ for all $\alpha$. Let $N(w)$ be a compact neighborhood of $w$. Then $N(w) \cap\left(\bigcup_{n=1}^{\infty} F^{n} y_{a} F^{n}\right) \neq \varnothing$ for all $\alpha$. There exist $t_{a}$, $s_{\alpha} \in F^{m}$ for some $m$ (same $m$ for both $t_{\alpha}, s_{\alpha}$ ) such that $t_{\alpha} y_{\alpha} s_{\alpha} \in N(w)$. By compactness of $D$, we can find subnets $t_{\beta}, y_{\beta}, s_{\beta}$, such that $t_{\beta} \rightarrow t, y_{\beta} \rightarrow z$, $s_{\beta} \rightarrow s$ and $t z s \in N(w)$. Since $t_{\beta} z s_{\beta} \in \bigcup_{n=1}^{\infty} F^{n} z F^{n}$, tzs $\in \overline{\bigcup_{n=1}^{\infty} F^{n} z F^{n}}$ so that $N(w) \cap\left(\overline{\bigcup_{n=1}^{\infty} F^{n} z F^{n}}\right) \neq \varnothing$. This means that $z \rightarrow w$. Q.E.D.

The following the orem is implicit in the results of [2] (cf. [2], proof of Theorem 2.5 and Theorem 2.4). We give the proof here for completeness.

Theorem 2.1. A state $y \in D$ is recurrent in the unilateral walks (and hence is in the kernel $K$ ) if and only if $\Sigma_{n} \mu^{n}\left(N(y) x^{-1}\right)=\infty$ for all open neighborhoods $N(y)$ of $y$ and some state $x \in D$.

Proof. We let $Z_{n}=X_{1} X_{2} \cdots X_{n}$ and $Z_{k}^{-1} Z_{n}=X_{k+1} X_{k+2} \cdots X_{n}$. In case $y$ is recurrent, then by [2] $P_{x}\left(Z_{n} \in N(y)\right.$ i.o. $)=1$ for every $x$ in the recurrence class $y D$. Next, suppose $\Sigma \mu^{n}\left(N(y) x^{-1}\right)=\infty$ for all $N(y)$ and some $x \in D$. Let $k$ be arbitrary positive integer. Then

$$
\sum_{j=1}^{k} \sum_{i=0}^{\infty} P_{x}\left(Z_{j+i k} \in N(y)\right)=\infty \text {. }
$$

So we can find an integer $m$ such that $1 \leq m \leq k$ and $\sum_{i=0}^{\infty} P_{x}\left(Z_{m+i k} \in N(y)\right)=\infty$. Now

$$
\begin{aligned}
1 & \geq P_{x}\left(Z_{n} \in N(y) \text { finitely often }\right) \\
& \geq \sum_{i=0}^{\infty} P_{x}\left(Z_{m+i k} \in N_{y}, Z_{n} \notin N_{y} \text { for all } n \geq m+(i+1) k\right) \\
& \geq \sum_{i=0}^{\infty} P_{x}\left(Z_{m+i k} \in N_{y}, Z_{m+i k}^{-1} Z_{n} \notin N_{y}^{-1} N_{y} \text { for all } n \geq m+(i+1) k\right) \\
& =P\left(Z_{n} \notin N_{y}^{-1} N_{y} \text { for all } n \geq k\right) \sum_{i=0}^{\infty} P_{x}\left(Z_{m+i k} \in N(y)\right)
\end{aligned}
$$

which means that, for any open set $N_{y}=N(y)$ containing $y$ and every positive integer $k$,

$$
P\left(Z_{n} \notin N_{y}^{-1} N_{y} \text { for all } n \geq k\right)=0 .
$$

(Here $N_{y}^{-1} N_{y} \equiv\left\{s ; z s \in N_{y}\right.$ for some $\left.z \in N_{y}\right\}=\bigcup\left\{z^{-1} N_{y} ; z \in N_{y}\right\}$.) Now given an open set $N$ containing $y$, by [2, Lemma 2.1], we can find an open set $N_{y}$ containing $y$ such that $N_{y}^{-1} N_{y} \subset y^{-1} N$. Then 


$$
\begin{aligned}
P_{y}\left(Z_{n} \in N \text { finitely often }\right) & \leq P\left(Z_{n} \in y^{-1} N \text { f.o. }\right) \leq P\left(Z_{n} \in N_{y}^{-1} N_{y} \text { f.o. }\right) \\
& =\sum_{i=1}^{\infty} P\left(Z_{i} \in N_{y}^{-1} N_{y}, Z_{n} \notin N_{y}^{-1} N_{y} \text { for all } n>i\right)=0 .
\end{aligned}
$$

Hence $P_{y}\left(Z_{n} \in N\right.$ i.o. $)=1$ and $y$ is recurrent and belongs to $K$ by [2]. Q.E.D.

3. The orem 3.1. $x$ is essential in the bilateral walk if and only if $P_{x}\left(W_{n} \in N(x)\right.$ i.o. $)=1$ for all $N(x)$ of $x$.

Proof. Suppose $P_{x}\left(W_{n} \in N(x)\right.$ i.o. $)=1$ for all $N(x)$. Then we claim that $x$ is essential and $x \in K$. Suppose $x \notin K$. Then there is $y \in \overline{\bigcup_{n=1}^{\infty} F^{n} x F^{n}}$ such that $x \notin \overline{\bigcup_{n=1}^{\infty} F^{n} y F^{n}}$. By using compactness of $D$, we can find $N(x)$, $N(y)$ such that $N(x) \cap\left(\bigcup_{n=1}^{\infty} F^{n} N(y) F^{n}\right)=\varnothing$ and $N(y) \cap\left(F^{k} x F^{k}\right) \neq \varnothing$ for some $k$. Now

$$
\begin{aligned}
0 & \leq P\left(X_{-k} \cdots X_{-1} x X_{1} \cdots X_{k} \in N(y)\right) \\
& =P\left(X_{-n} \cdots X_{-1} x X_{1} \cdots X_{n} \in N(x) \text { i.o., } X_{-k} \cdots X_{-1} x X_{1} \cdots X_{k} \in N(y)\right) \\
& =\int_{N(y)} P\left(X_{-n} \cdots X_{-1} x X_{1} \cdots X_{n} \in N(x)\right. \text { i.o.l } \\
& \left.\quad X_{-k} \cdots X_{-1} x X_{1} \cdots X_{k}=Z\right) \mu^{k} *{ }_{x}\left(\mu^{k}\right)(d z) \\
& =\int_{N(y)} P\left(X_{-n} \cdots X_{-1} z X_{1} \cdots X_{n} \in N(x) \text { i.o. }\right) \mu^{k} *{ }_{x}\left(\mu^{k}\right)(d z) \\
& =0 \text { since } N(x) \cap\left(F^{n} N(y) F^{n}\right)=\varnothing \text { for all n. }
\end{aligned}
$$

The above contradiction proves that $x \in K$ and therefore $x$ is essential.

Conversely, súppose $x \in K$. Then for any $y \in \overline{\bigcup_{n=1}^{\infty} F^{n} x F^{n}}, x \in$ $\overline{\bigcup_{n=1}^{\infty} F^{n} y F^{n}}$. Let $U$ be an open neighborhood of $x$. Then $U \cap\left(F^{n} y F^{n}\right) \neq \varnothing$ for some $n$. Let $U_{n}=\left\{y \in \overline{\bigcup_{n=1}^{\infty} F^{n} x F^{n}} ; U \cap F^{n} y F^{n} \neq \varnothing\right\}$. Clearly the $U_{n}$ 's are relatively open in $\overline{\bigcup_{n=1}^{\infty} F^{n} x F^{n}}$. (F or the support of $P_{b}^{n}(y, \cdot)=$ $\mu^{n} *\left[\mu^{n}\left(y^{-1} \cdot\right)\right](\cdot)$ in $F^{n} y F^{n}$. It follows that $U_{n}=\left\{y \in \overline{U F^{n} x F^{n}} ; P_{b}^{n}(y, U)>\right.$ $0\}$. But by Lemma 2.2, $P_{b}^{n}(\cdot, U)$ is lower semicontinuous so $U_{n}$ are relatively open.) By compactness, there is a finite subcover and for some finite $m$,

$$
g(y)=\sum_{1 \leq j \leq m} \mu^{j} *{ }_{y}\left(\mu^{j}\right)(U)>0 \text { for all } y \in \bigcup_{n=1}^{\infty} F^{n} x F^{n} .
$$

By lower semicontinuity of $g(y)$ (Lemma 2.2 ), there is $p>0$ such that $g(y) \geq p$ for all $y \in \overline{\bigcup_{n=1}^{\infty} F^{n} x F^{n}}$. Let $q=p / m$. Now $P\left(X_{-k} \cdots X_{-1} z X_{1} \cdots X_{k} \notin U, 1 \leq k \leq m\right) \leq 1-q$ for all $z \in \overline{\bigcup_{n=1}^{\infty} F^{n} x F^{n}}$. 
Also for any positive integer $s$,

$$
\begin{aligned}
P & \left(X_{-k} \cdots X_{-1} x X_{1} \cdots X_{k} \notin U, m s<k \leq m(s+1)\right) \\
& =\int P\left(X_{-k} \cdots X_{-1} x X_{1} \cdots X_{k} \notin U, m s<k \leq m(s+1)\right. \\
& \left.X_{-m s} \cdots X_{-1} x X_{1} \cdots X_{m s}=z\right) \mu^{m s} * x_{x}\left(\mu^{m s}\right)(d z) \\
& =\int P\left(X_{-k} \cdots X_{-1} z X_{1} \cdots X_{k} \notin U, 1 \leq k \leq m\right) \mu^{m s} *{ }_{x}\left(\mu^{m s}\right)(d z) \\
& \leq 1-q .
\end{aligned}
$$

Now setting $W_{k}=X_{-k} \cdots X_{-1} x X_{1} \cdots X_{k}$ we have

$$
\begin{aligned}
& P\left(X_{-k} \cdots X_{-1} x X_{1} \cdots X_{k} \notin U, m s<k \leq m(s+2)\right) \\
& =P\left(W_{k} \notin U, m(s+1)<k \leq m(s+2) \mid W_{k} \notin U, m s<k \leq m(s+1)\right) \\
& \times P\left(W_{k} \notin U, m s<k \leq m(s+1)\right) \\
& =P\left(W_{k} \notin U, m(s+1)<k \leq m(s+2) \mid W_{m(s+1)} \notin U\right) \\
& \times P\left(W_{k} \notin U, m s<k \leq m(s+1)\right) \\
& =\frac{1}{\mu^{m(s+1)} * x_{x}^{\left(\mu^{m(s+1)}\right)(\tilde{U})}} \int_{\tilde{U}} P\left(X_{-k} \cdots X_{-1} z X_{1} \cdots X_{k} \notin U, 1 \leq k \leq m\right) \\
& \cdot \mu^{m(s+1)} * x_{x}\left(\mu^{m(s+1)}\right)(d z)
\end{aligned}
$$$$
\times P\left(W_{k} \notin U, m s<k \leq m(s+1)\right)
$$$$
\leq(1-q)^{2}
$$

Similarly, $P\left(W_{k} \notin U, m s<k<m(s+i)\right) \leq(1-q)^{i}$, which means that $P\left(W_{k} \in U\right.$ f.o. (finitely often $\left.)\right)=0$. Hence $P\left(W_{k} \in U\right.$ i.o. $)=1$. Q.E.D.

Theorem 3.2. $x$ is recurrent in the bilateral walk if and only if $\sum_{n=1}^{\infty} \mu^{n} *{ }_{x}\left(\mu^{n}\right)(N(x))=\infty$ for all neighborboods $N(x)$ of $x$.

Proof. The " $\Rightarrow$ " part is trivial by the Borel-Cantelli lemma. For the converse, suppose $\Sigma_{n} \mu^{n} *{ }_{x}\left(\mu^{n}\right)(N(x))=\infty$ for all $N(x)$ of $x$. We claim that $x \in K$. If $x \notin K$, then for $y \in D$, there would exist a neighborhood $N_{y}(x)$ of $x$ such that $\Sigma_{n} \mu^{n}\left(N_{y}(x) y^{-1}\right)<\infty$; this is because by Theorem 2.1, if $\Sigma \mu^{n}\left(N(x) y^{-1}\right)=\infty$ for all $N(x), x, y \in D$, then $x \in K$. Now there exist $N_{y}^{\prime}(x)$, $N(y)$ such that $N_{y}^{\prime}(x) N(y)^{-1} \subset N_{y}(x) y^{-1}$ by [2, Lemma 2.1]. (One uses a compactness argument to prove this result.) Since $D$ is compact, there exist $N\left(y_{i}\right), 1 \leq i \leq n$, such that $D \subset \bigcup_{i=1}^{n} N\left(y_{i}\right)$. If $N_{0}(x)$ (= a compact neighborhood 
of $x) \subset \bigcap_{i=1}^{n} N_{y_{i}}^{\prime}(x), \Sigma \mu^{n}\left(N_{0}(x) N\left(y_{i}\right)^{-1}\right)<\infty, 1 \leq i \leq n$, so that $\Sigma \mu^{n}\left(N_{0}(x) D^{-1}\right)<\infty$. But

$$
\begin{aligned}
\infty & =\sum_{n=1}^{\infty} \mu^{n} *_{x}\left(\mu^{n}\right)\left(N_{0}(x)\right)=\sum_{n=1}^{\infty} \int \mu^{n}\left(N_{0}(x) z^{-1}\right){ }_{x}\left(\mu^{n}\right)(d z) \\
& \leq \sum_{n=1}^{\infty} \mu^{n}\left(N_{0}(x) z_{n}^{-1}\right)<\infty .
\end{aligned}
$$

where

$$
\mu^{n}\left(N_{0}(x) z_{n}^{-1}\right)=\max _{z \in D} \mu^{n}\left(N_{0}(x) z^{-1}\right) .
$$

This maximum is attained since $D$ is compact and the mapping $z \rightarrow$ $\mu^{n}\left(N_{0}(x) z^{-1}\right)$ is upper semicontinuous. (Since $N_{0}(x)$ is compact, $D-N_{0} \equiv$ $N_{0}^{c}$ is open so $\mu^{n}\left(N_{0} z^{-1}\right)=\mu^{n}\left(\left(D-N_{0}^{c}\right) z^{-1}\right)=1-\mu^{n}\left(N_{0}^{c} z^{-1}\right)$ and $-\mu^{n}\left(N_{0}^{c} z^{-1}\right)$ is upper semicontinuous by Lemma 2.2.) The above contradiction proves that $x \in K$ and so $x$ is recurrent in the bilateral walk. Q.E.D.

Theorem 3.3. Let $x \in K$. Then

(i) For all $N(x), \overline{\lim } \mu^{n}(N(x))>0$.

(ii) For all $N(x), \varlimsup \lim \mu^{n} *_{x}\left(\mu^{n}\right)(N(x))>0$.

Proof. (i) First, let $U$ be any open set containing $K=$ the kernel of $D$. We know (by $\left[4\right.$, p. 141]) that $\mu^{n}(U) \rightarrow 1$ as $n \rightarrow \infty$. Now suppose for all $x \in K$, there is $N(x)$ such that $\overline{\lim }_{n \rightarrow \infty} \mu^{n}(N(x))=0$. Then since $K$ is compact, $K \subset U=\bigcup_{i=1}^{k} N\left(x_{i}\right)$, for some $k$ and $\overline{\lim } \mu^{n}\left(N\left(x_{i}\right)\right)=0$; but this implies $\varlimsup \lim \mu^{n}(U)=0$, which is a contradiction. Hence there is $x \in K$ such that $\left\lceil\mu^{n}(N(x))>0\right.$ for all $N(x)$. Next, let $I=\left\{x \in D ; \varlimsup \lim \mu^{n}(N(x))>0\right.$ for all $N(x)\}$. $I \neq \varnothing$ since $x \in I \cap K$. Let $z \in D$. Then, for all $N(x z)$, there exist $N(x), N(z)$ such that $N(x) N(z) \subset N(x z)$. Now, choosing $k$ so that $\mu^{k}(N(z))>$ 0 , we have

$$
\mu^{n+k}(N(x z)) \geq \int_{N(z)} \mu^{n}\left(N(x z) w^{-1}\right) \mu^{k}(d w) \geq \mu^{n}(N(x)) \mu^{k}(N(z)),
$$

since for all $w \in N(z), N(x z) w^{-1} \supset N(x)$; this means that $\overline{\lim } \mu^{n}(N(x z))>0$. Similarly, $\overline{\lim } \mu^{n}(N(z x))>0$. Hence $I$ is an ideal and hence $K \subset I$.

(ii) We have $\mu^{n} *{ }_{x}\left(\mu^{n}\right)(N(x))=\int \mu^{n}\left(N(x) y^{-1}\right)_{x}\left(\mu^{n}\right)(d y)$. Now, given $N(x)$, we can find $N_{1}(x)$ such that $N_{1}(x) x^{-1} \subset N(x) y^{-1}$ for all $y \in N_{1}(x)$; ([proof] for otherwise, there would exist a net $y_{a} \rightarrow x$ such that $y_{a} \in N_{1}(x)$ and $N_{1}(x) x^{-1} \cap\left(N(x) y_{a}^{-1}\right)^{\sim} \notin \varnothing$, so that we can find $z_{a} \in N_{1}(x) x^{-1} \cap$ $\left(N(x) y_{a}^{-1}\right)^{\sim}$ such that $z_{a} x \in N_{1}(x), z_{a} y_{a} \notin N(x)$, which gives a contradiction (by using compactness of $D$ )). Hence 


$$
\begin{aligned}
\mu^{n} *{ }_{x}\left(\mu^{n}\right)(N(x)) & \geq \int_{N_{1}(x)} \mu^{n}\left(N_{1}(x) s^{-1}\right) \\
x & \left(\mu^{n}\right)(d s) \\
& =\mu^{n}\left(N_{1}(x) x^{-1}\right) \mu^{n}\left(x^{-1} N_{1}(x)\right),
\end{aligned}
$$

which shows that $\varlimsup_{\lim } \mu^{n} *_{x}\left(\mu^{n}\right)(N(x))>0$. Q.E.D.

Concluding remarks. (i) If $S$ is completely simple and discrete, then Larisse [1] proved that the number of essential classes is 1 or 2 for the bilateral random walk. Now if there is a recurrent element, these classes are recurrent by [1]. By [6, A remark on invariant measures $]$, there is an invariant measure $\nu_{i}$ on each recurrent class of $D=K$. It can be checked, that an invariant (stationary) measure for the bilateral walk is also an invariant measure for the unilateral walks. Then the sum of these $\nu_{i}(i \leq 2)$ measures would be an invariant measure for the three walks on the whole $D$, since $\int_{C} P_{b}(x, N)\left(\nu_{1}+\nu_{2}\right)(d x)=\nu_{1}(N)$, since the support of the invariant measure $\nu_{1}$ on the class $C_{1}$ is contained in $C_{1}$ (see [6]).

(ii) We have imposed 2 nd countability as a blanket assumption in order to insure that certain sets and the $W_{n}$ 's are measurable $\left(D^{\infty}, P\right.$ ) (cf. Lemma 2.1). We can take $\left(D^{\infty}, P\right)$ to be the Bledsoe-Morse product measure extension of the usual product measure space as utilized in R. M. Dudley, Pathological topologies and random walks on abelian groups, Proc. Amer. Math. Soc. 15 (1964), 231-238; see particularly pp. 233, 234. Then the $W_{n}$ would be measurable (Borel) as functions on $\left(D^{\infty}, P\right)$. First countability was used in Lemma 2.2 (where sequences were used). But one can proceed as for proving that $\mu *{ }_{x} \mu(N)$ is Borel measurable following the argument in [4, p. 130] using double integral (instead of single); then one may use

$$
\mu^{2} *{ }_{x}\left(\mu^{2}\right)(N)=\int_{\mu *{ }_{y}}(\mu)(N) \mu *{ }_{x}(\mu)(d y)
$$

and compactness to prove that $\mu^{2} *{ }_{x}\left(\mu^{2}\right)(N)$ is lower semicontinuous.

(iii) The techniques and methods used by the authors to tackle recurrence problems in bilateral walks have been remarkedly different from those in the case of one-sided walks [2], [6] and also from those of Larisse who had the tools of Markov chain at his disposal. By studying Larisse's works [1] on countable discrete semigroups, the authors find it reasonable to guess that even in the case of general locally compact groups, an element $x$ in $D$ is recurrent in the bilateral walk iff it is recurrent in the one-sided walk. In the case of locally compact abelian groups, we observe the following. In such a group let $R^{*}=\left\{x \in D ; P\left(W_{n} \in N_{x}\right.\right.$ i.o. $)=1$ for all neighbor- 
hoods $N_{x}$ of $\left.x\right\}$. Then if $R^{*} \neq \varnothing$, by $\mathrm{R}$. M. Loynes, Products of independent random elements in a topological group, $\mathrm{Z}$. Wahrscheinlichkeitstheorie 1 (1962/63), 446-455, the unilateral walks are recurrent and $D=a_{\text {. group. }}$ Using Loynes' arguments applied to $W_{n}$ in the form $X_{-1} X_{-2} \cdots$ $X_{-n} X_{0} X_{n} X_{n-1} \cdots X_{1}$ one can easily show that $y \in R^{*}$ and $c \in D$ implies $c^{-1} y c^{-1} \in R^{*}$. Hence for any $y \in R^{*}$ and any $c \in D$ we have $y^{-1} \in R^{*}$ and $c^{2} y \in R^{*}$; more over $\left(\bigcup_{k=1}^{\infty} F^{2 k}\right) R^{*} \subset R^{*}$.

Added in proof. The essential classes for $W_{n}$ above are at most two. To see this, first let $\mu^{2 n} \rightarrow \beta$. If $K=X \times G \times Y^{n}$ is the kernel of $D$, then $F S_{\beta}=S_{\beta} F, F^{2} S_{\beta}=S_{\beta}$ where $S_{\beta}$ (the support of $\beta$ ) is $X \times G_{1} \times Y\left(G_{1}\right.$ a subgroup of $G$ ). The essential classes of the bilateral walk induced by $\mu * \beta$ are seen to be the same as those of the original walk (since $\mu^{2 n} \rightarrow \beta$ ). But the se are of the form $A_{x}=\overline{U\left(F S_{\beta}\right)^{n} x\left(F S_{\beta}\right)^{n}}, x \in K$. If $x \in S_{\beta}, A_{x}=$ $\overline{U F^{n} S_{\beta} F^{n}}$ and if $y, z \in K-S_{\beta}$, then $S_{\beta} y S_{\beta}=S_{\beta} z S_{\beta}$ and so $A_{y}=A_{z}$. Second, let $\mu^{2 n}$ not converge. Then by [4, p. 152], there is a normal subgroup $G^{\prime}$ of $G$ with $\left(X \times G^{\prime} \times Y\right) F=X \times g G^{\prime} \times Y, g \notin G^{\prime}$, and $\overline{U_{g}^{n} G^{\prime}}=G$. Here $X \times G^{\prime} \times Y$ is the support of $Q$, the identity of the kernel group of $\left\{\mu^{n}: n \geq 1\right\}^{-}$. Then as above, the essential classes of the bilateral walk induced by $\mu * Q$ are at most two and are the same as those of the original walk since $Q$ is a cluster point of $\left\{\mu^{n}\right\}$.

\section{REFERENCES}

1. J. Larisse, Marches au hasard sur les demigroupes discrets. I, II, Ann. Inst. H. Poincaré 8 (1972), 107-174.

2. A. Mukherjea, T. C. Sun and N. A. Tserpes, Random walks on compact semigroups, Proc. Amer. Math. Soc. 39 (1973), 599-605.

3. M. Rosenblatt, Transition probability operators, Proc. Fifth Berkeley Sympos. Math. Statist. and Probability (Berkeley, Calif., 1965/66), Vol. II: Contributions to Probability Theory, Part 2, Univ. California Press, Berkeley, Calif., 1967, pp. 473-483. MR $35 \# 3742$.

4. - Markov processes: Structure and asymptotic behavior, Die Grundlehren der math. Wissenschaften, Band 184, Springer-Verlag, New York, 1971.

5. - Stationary measures for random walks on semigroups, Semigroups (Proc. Sympos., Wayne State Univ., Detroit, Mich., 1968), Academic Press, New York, 1969, pp. 209-220. MR 41 \#4666.

6. T. C. Sun, A. Mukherjea and N. A. Tserpes, On recurrent random walks on semigroups, Trans. Amer. Math. Soc. 185 (1973), 213-227.

DEPARTMENT OF MATHEMATICS, UNIVERSITY OF SOUTH FLORIDA, TAMPA, FLORIDA 33620 